\title{
Chemsex Among Men Who Have Sex With Men: a Sexualized Drug Use Survey Among Clients of the Sexually Transmitted Infection Outpatient Clinic and Users of a Gay Dating App in Amsterdam, the Netherlands
}

\author{
Susanne Drückler, MSc, * Martijn S. van Rooijen, MSc, * and Henry J.C. de Vries, MD, PhD*广ł
}

\begin{abstract}
Objectives: Chemsex (i.e., drug use during sex) is practiced by some men who have sex with men (MSM) and is associated with high-risk behavior. In a cross-sectional study at the sexually transmitted infection (STI) clinic of Amsterdam, we explored chemsex practices, risk behavior, and STI prevalence. Method: A survey on chemsex ( $\gamma$-hydroxybutyrate, crystal methamphetamine, and/or mephedrone) was offered to clinic clients during routine STI screening and to Amsterdam users of a gay online dating app. Associations were assed using $\chi^{2}$ test and multivariable regression.

Results: Chemsex in the past 6 months was practiced by $866(17.6 \%)$ of 4925 MSM clients and by 159 (1.5\%) of 10857 non-MSM clients. Among gay dating app users, the proportion that reported chemsex engagement was higher than among MSM visiting the STI clinic (29.3\% [537/1832] vs. $17.6 \% ; P<0.001)$. Chemsex was a significant risk factor for bacterial STI in HIV-negative MSM visiting the STI clinic (adjusted odd ratio, 1.5; 95\% confidence interval, 1.2-1.8), but not in HIV-positive MSM. A majority practiced chemsex once a month or less, and $87.0 \%$ reported sex without drug use in the past month.
\end{abstract}

Conclusions: In Amsterdam, chemsex is frequently practiced and significantly associated with bacterial STI in HIV-negative MSM but not in

From the *Public Health Service of Amsterdam, Department of Infectious Diseases; and $\dagger$ Department of Dermatology and $\ddagger$ Amsterdam Infection and Immunity Institute (AI\&II), Academic Medical Centre, University of Amsterdam, Amsterdam, the Netherlands

Acknowledgments: The authors thank Michelle Kroone for setting up the database and Bart-Jan Mulder and Elske Marra for the support in developing the online survey.

Conflicts of Interest and Source of Funding: None declared and no funding received.

Contributors: S. Drückler, M. van Rooijen, and H.J.C. de Vries designed the study protocol. S. Drückler performed the statistical analysis. Results were thoroughly discussed by S. Drückler, M. van Rooijen, and H.J.C. de Vries. S. Drückler drafted the manuscript, all authors commented on draft versions, and all approved the final version.

Correspondence: Henry J.C. de Vries, MD, PhD, Public Health Service of Amsterdam, Department of Infectious Diseases, STI Clinic, PO Box 2200, 1000 CE Amsterdam, the Netherlands. E-mail: h.j. devries@amc.uva.nl; Susanne Drückler, MSc, Public Health Service of Amsterdam, Cluster of Infectious Diseases, Department of Research, PO Box 2200, 1000 CE Amsterdam, the Netherlands. E-mail: sdruckler@ggd.amsterdam.nl.

Received for publication July 26, 2017, and accepted October 28, 2017.

Copyright (C) 2017 The Author(s). Published by Wolters Kluwer Health, Inc. on behalf of the American Sexually Transmitted Diseases Association. This is an open-access article distributed under the terms of the Creative Commons Attribution-Non Commercial-No Derivatives License 4.0 (CCBY-NC-ND), where it is permissible to download and share the work provided it is properly cited. The work cannot be changed in any way or used commercially without permission from the journal.

DOI: 10.1097/OLQ.0000000000000753
HIV-positive MSM. Future prevention strategies to reduce STI incidence should especially target HIV-negative MSM engaging in chemsex.

$\mathrm{D}$ rug use in general and before or during sex is highly prevaent among men who have sex with men (MSM). ${ }^{1,2}$ In 2010, a large European MSM Internet Survey in 44 European cities showed that drug use was particularly prevalent in Amsterdam. ${ }^{3}$ Associations between drug use and sexual risk behavior among MSM have frequently been reported. ${ }^{3-6}$ However, less research focused on the phenomenon called "chemsex," which is a phenomenon frequently referred to on gay networking apps to identify those using and seeking recreational drugs during sex. ${ }^{7}$ A stricter definition of chemsex is "the use of any combination of drugs including crystal methamphetamine, mephedrone, and/or gamma hydroxybutyrate (GHB), before or during sex by men who have sex with men (MSM).",

Positive effects and motivations of chemsex engagement are often described by users as intense sexual arousal, increased libido, and enhanced performance. ${ }^{8,9}$ Chemsex is highly associated with sexual risk-taking behavior, physical and mental health consequences, and possible addiction. ${ }^{2,8,10}$ Different studies demonstrated that chemsex engagement was associated with diagnosed and self-reported sexually transmitted infections (STIs). ${ }^{8,11,12}$

In addition, there are signals of a perceived increase in chemsex in areas with a large MSM population, ${ }^{8}$ mostly in England, ${ }^{13,14}$ but also in the Netherlands. ${ }^{15}$ To identify clients with problematic drug use that should be offered additional help, it is advocated to enquire after recreational drug use and chemsex as part of routine consultations in sexual health care settings and during health promotion/prevention..$^{3,6,7,10,12,16}$ Therefore, as of July 2016, the Amsterdam STI clinic started to routinely collect data about chemsex engagement in all clients. To investigate the representativeness of our population, the same survey was offered to Amsterdam users of a popular gay dating app. The aim of the present study is to quantify the proportion of STI clinic clients engaging in chemsex and to identify whether chemsex is a risk factor for an STI diagnosis. We hypothesized that chemsex is a predictor for bacterial STI.

\section{MATERIALS AND METHODS}

\section{Study Population}

STI Clinic. The STI outpatient clinic in Amsterdam is the largest center for STI care in the Netherlands, with up to 40,000 consultations each year. ${ }^{17} \mathrm{We}$ test and treat (free of charge) patients who are younger than 25 years, are commercial sex workers, are MSM, are notified of an STI by a sex partner, are of non-West 
European or non-North American origin, have STI-related symptoms, or have a partner from an STI endemic region.

We retrieved anonymized data from the electronic patient files of all clients of the STI clinic between July 21, 2016, and December 31, 2016. A first visit of a client was included in the present study and repeated consultations in the same client were excluded. Because all data were anonymous and the clinical data were routinely collected, ethical approval was deemed unnecessary for this study.

Dating App Online Survey. In addition, users of an online dating app (Grindr) were offered a chemsex survey for 2 days during the period of the Europride festival in the summer of 2016 in Amsterdam. All users of the app in the area of Amsterdam during those 2 days received a push message on their app and were asked to fill in the survey.

\section{Demographics, Sexual Behavior, and Chemsex}

Besides routinely collected data from the electronic patient files of the STI clinic (age, ethnicity [ethnicity was defined according to Statistics Netherlands on the basis of country of birth, maternal and paternal country of birth], ${ }^{18}$ reason of visit, HIV status, sex of sex partner, condom use during anal sex, number of sex partners), health care workers collected data on the use of crystal methamphetamine, GHB, and/or mephedrone during sex in the past 6 months. Chemsex was defined as the use of at least one of the previously mentioned drugs during or before sex. ${ }^{7}$ Chemsex characteristics recorded were as follows: frequency of chemsex engagement; injecting drugs and, if so, if needles were shared; last time having sex without any of the previously mentioned drugs (sober sex), and condomless receptive and/or insertive anal sex after using one of the drugs.

The same questions regarding chemsex were asked in the chemsex survey to users of the online dating app. No questions regarding demographics, risk-taking behavior, or STI history were asked in this study population.

\section{Testing Procedure at the STI Clinic}

Clients were tested for Chlamydia trachomatis (Ct; including lymphogranuloma venereum [LGV] in case anorectal $\mathrm{Ct}$ was found), Neisseria gonorrhoeae (Ng), and syphilis. Urethral Gram stain analysis was performed only in male patients with urogenital signs or symptoms (discharge, painful and/or frequent urination). In those with more than 10 polymorph nucleated leucocytes per high-power field in a urethral Gram-stained smear and no infection with $\mathrm{Ct}$ and $\mathrm{Ng}$, a definitive diagnosis of nonspecific urethritis (NSU) was made. ${ }^{19}$ All MSM who had not previously tested HIV positive were tested for HIV unless they actively opted out.

Urine and pharyngeal and rectal specimens were tested for both $\mathrm{Ct}$ and $\mathrm{Ng}$ using the Aptima Combo 2 assay (Hologic, Marlborough, MA). In asymptomatic MSM, both urine and rectal specimens were self-collected, whereas in symptomatic MSM, medical staff collected rectal specimens. Medical staff collected pharyngeal specimens in all MSM. HIV antibodies were tested using a rapid HIV test (Alere Determine HIV-1/2 antibody test; Medical Co., Ltd.; Chiba, Japan) and the HIV Ab/Ag test (LIAISON XL; Diasorin, Saluggia, Italy), and syphilis serology was performed using the Treponema Screen (LIAISON XL; Diasorin). All bacterial STI diagnoses were based on laboratory outcomes of the STI consultation in which the chemsex questions were asked.

\section{Statistical Analysis}

Using the $\chi^{2}$ test for independence, we compared characteristics and STI positivity and epidemiologic characteristics of MSM who engaged in chemsex with those who did not. Using the $\chi^{2}$ test for independence and the Fisher exact test, we compared the specified chemsex patterns between men at the STI clinic and men who filled in the online survey. Univariable logistic regression analysis was performed to examine associations with a bacterial STI. A bacterial STI was defined as diagnosis with $\mathrm{Ct}$ (both non-LGV and LGV) and/or Ng and/or infectious syphilis (NSU not included). The association with the following variables was assessed: age, ethnicity, condomless anal sex (CAS), number of partners in the preceding 6 months, HIV status, notification of STI exposure, STI-related symptoms, and chemsex engagement. Age and the number of sex partners were divided into 4 categories based on interquartile range (IQR). HIV status was divided into HIV-negative MSM, including MSM who refused an HIV test, and HIV-positive MSM. All variables were entered into a multivariable logistic regression model using backward selection. We checked for interactions between chemsex and all other variables.

In addition, univariable analyses were performed to examine the association of chemsex engagement with a diagnosis of chlamydia, gonorrhea, and syphilis individually.

Data analyses were performed using SPSS package v21.0 (SPSS, Chicago, IL) and STATA software (STATA Intercooled version 13.0, College Station, TX). In the present study, $P<0.05$ was considered statistically significant.

\section{RESULTS}

\section{Characteristics of Study Population}

Between July 21, 2016, and December 31, 2016, 16,164 consultations in unique clients took place at the STI clinic of Amsterdam. Of those clients, 4925 (31.2\%) were among unique MSM in whom chemsex engagement was asked (Fig. 1).

Because chemsex engagement in non-MSM clients was rare $(1.5 \% ; \mathrm{n}=159)$ and only 6 clients $(3.8 \%)$ used crystal methamphetamine and 7 clients $(4.4 \%)$ used mephedrone, we did not further explore this group and confined further analyses on chemsex characteristics to MSM clients.

Of all MSM questioned about chemsex in this study period, 1049 (21.2\%) used crystal methamphetamine and/or GHB and/or mephedrone in the previous 6 months, and $866(17.6 \%)$ of all MSM reported the use of one of the drugs during sex (chemsex). In men who reported chemsex, most demographic variables differed significantly from those who did not report chemsex (Table 1).

Men who have sex with men who reported chemsex reported higher sexual risk behavior (more sex partners, more often CAS), reported more often STI-related symptoms and a notification of STI exposure, were more often on preexposure prophylaxis (PrEP) as participants of the Amsterdam PrEP research study ${ }^{20}$ ( $25.5 \%$ vs. $5.7 \% ; P<0.001)$, and had more often a bacterial STI diagnosis (chlamydia, gonorrhea, LGV, and/or infectious syphilis; $31.1 \%$ vs. $20.7 \% ; P<0.001)$. New HIV diagnosis ( $0.4 \%$ vs. $0.7 \%$; $P=0.26)$ did not differ significantly between both study groups.

\section{Associations With Bacterial STI Diagnosis}

In the univariable analyses, chemsex engagement was significantly associated with a diagnosis of chlamydia (odds ratio [OR], 1.6; 95\% confidence interval [CI], 1.3-1.9), gonorrhea $(\mathrm{OR}, 1.9 ; 95 \% \mathrm{CI}, 1.6-2.3)$, and syphilis $(\mathrm{OR}, 1.6 ; 95 \%$ CI, 1.1-2.4; results not shown in tables). In the univariable analysis using the global outcome variable bacterial STI, age, chemsex engagement in the past 6 months, HIV status, notification of STI exposure, STI-related symptoms, CAS, and the number of sex partners were significantly associated with a bacterial STI diagnosis (Table 2). 


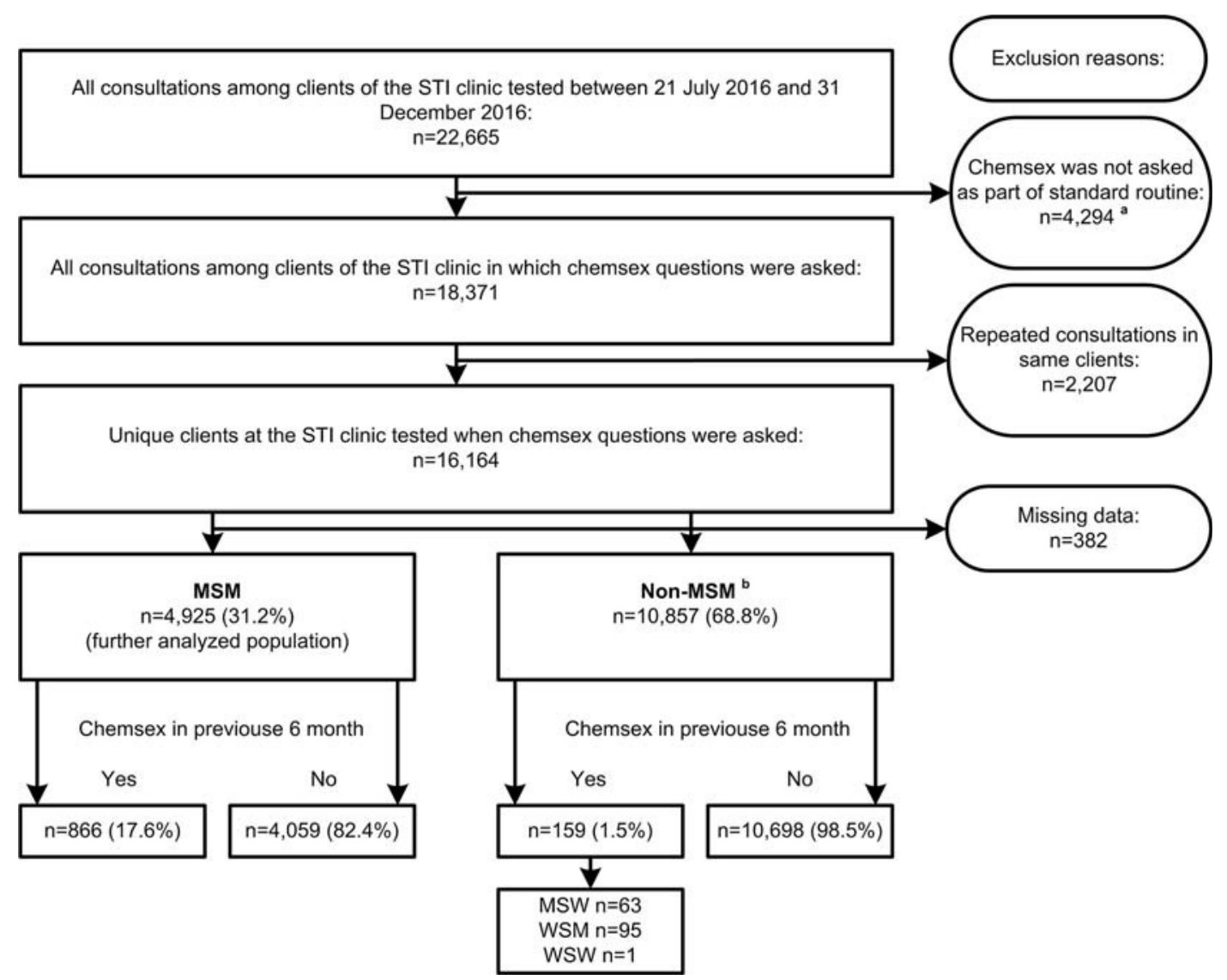

Figure 1. Flowchart of all STI clinic consultations in which chemsex practices were asked at the Public Health Service of Amsterdam, the Netherlands; July to December 2016. ${ }^{\text {a}}$ Chemsex characteristics were not asked when someone was tested: (1) through the online MSM screening program (Man Tot Man), (2) through participation of the Amsterdam Cohort Study, (3) as part of a sexual health (SENSE) consultation for clients till the age of 25 years without STI screening, and (4) through a self-screening test (not applicable to MSM).

${ }^{b}$ Non-MSM are defined as men who have sex with women (MSW), women who have sex with men (WSM), and women who have sex with women (WSW).

In the multivariable analyses, the interaction between HIV status and chemsex was significant. HIV-negative MSM who reported chemsex had a significant higher risk of a bacterial STI than did HIV-negative MSM who did not report chemsex (adjusted OR, 1.5; 95\% CI, 1.2-1.8). An additional analysis with the same multivariable model, using HIV-positive men not engaging in chemsex as a reference category, showed that there was no significant difference in HIV-positive men, irrespective of chemsex engagement.

\section{Pattern of Chemsex}

Of the 866 MSM who engaged in chemsex in the past 6 months, GHB was the most reported drug used (93.0\%; Table 3). Crystal methamphetamine was used by 190 (22.1\%) MSM, and mephedrone was used by 137 (16.0\%) MSM. Regardless of the drug, most MSM reported the use of the drug once per month or less (GHB, 62.7\%; crystal methamphetamine, 75.3\%; mephedrone, $82.5 \%)$. Most of the men $(74.7 \%)$ used only 1 of the 3 drug in the past 6 months (GHB, 68.1\%; crystal methamphetamine, $4.2 \%$; and mephedrone, $2.4 \%$ ). A smaller group, $19.4 \%$ of the men, used 2 of the 3 drugs and $5.8 \%$ reported the use of all 3 drugs.

Among MSM who reported chemsex, 52 (6.1\%) disclosed injecting drugs in the past 6 months, of whom 1 person shared needles. Most MSM (87.0\%) reported sober sex in the past months, whereas for the rest of the men (13.0\%), it was longer than 3 months ago that they had sex without the use of 1 of the 3 drugs. Lastly, $69.8 \%$ of the MSM who reported chemsex disclosed CAS (active/ passive) while using one of the drugs.

Of all men $(\mathrm{n}=1832)$ who participated in the online survey, $29.3 \%$ (537) reported chemsex in the past 6 months. Chemsex engagement differed significantly between STI clinic clients and men who filled in the online survey $(17.6 \%$ vs. $29.3 \% ; P<0.001)$. There was no significant difference regarding GHB and crystal methamphetamine use between STI clinic clients and online participants who engaged in chemsex. Mephedrone use was reported more often in the online survey group than at the STI clinic $(28.3 \%$ vs. $16.0 \% ; P<0.001)$. Compared with the clinic population, less online participants had sober sex in the past month $(87.0 \%$ vs. $76.8 \% ; P<0.001)$ and less condomless anal chemsex $(69.8 \%$ vs. $62.4 \% ; P=0.005)$.

\section{DISCUSSION}

Here we show that $17.6 \%$ of all MSM visiting the STI clinic of Amsterdam had engaged in chemsex in the previous 6 months. Chemsex among this group was found to be significantly associated with a bacterial STI diagnosis. This association remains significant in the group of HIV-negative MSM after adjusting for demographics and high-risk sexual behavior. However, in HIVpositive men, after adjusting, there is no significant difference in the risk of a bacterial STI diagnosis between those engaging 
TABLE 1. Demographics, Consultation Characteristics, STI Diagnosis, and New HIV Diagnosis Among 866 MSM Practicing Chemsex and 4059 MSM not Practicing Chemsex at the STI Clinic, Public Health Service of Amsterdam, the Netherlands; July to December 2016

\begin{tabular}{|c|c|c|c|c|}
\hline Variables & $\begin{array}{c}\text { Chemsex, } \\
\text { Yes }(n=866), n(\%)\end{array}$ & $\begin{array}{c}\text { Chemsex, } \\
\text { No }(n=4059), n(\%)\end{array}$ & $\boldsymbol{P}$ & $\begin{array}{c}\text { All MSM } \\
(n=4925), n(\%)\end{array}$ \\
\hline Median age (IQR), y & $37(29-48)$ & $34(26-45)$ & $<0.001$ & $35(27-46)$ \\
\hline \multicolumn{5}{|l|}{ Age categories, $\mathrm{y}$} \\
\hline$\leq 27$ & $167(19.3)$ & $1212(29.9)$ & $<0.001$ & $1379(28.0)$ \\
\hline $28-35$ & $210(24.2)$ & $998(24.6)$ & & $1208(24.5)$ \\
\hline $36-46$ & $250(28.9)$ & $957(23.6)$ & & $1207(24.5)$ \\
\hline$\geq 47$ & $239(27.6)$ & $892(22.0)$ & & $1131(23.0)$ \\
\hline \multicolumn{5}{|l|}{ Ethnicity* } \\
\hline Western & $600(69.3)$ & $2644(65.1)$ & 0.020 & $3244(65.9)$ \\
\hline Non-Western & $266(30.7)$ & $1415(34.9)$ & & $1681(34.1)$ \\
\hline HIV status $\dagger$ & & & $<0.001$ & \\
\hline HIV-positive & $298(34.4)$ & $653(16.1)$ & & $951(19.3)$ \\
\hline HIV-negative & $568(65.6)$ & $3406(83.9)$ & & $3974(80.7)$ \\
\hline \multicolumn{5}{|l|}{ Reason of consultation } \\
\hline Notification of STI exposure & $266(30.7)$ & $878(21.6)$ & $<0.001$ & $1144(23.2)$ \\
\hline STI-related symptoms & $218(25.2)$ & $869(21.4)$ & 0.015 & $1087(22.1)$ \\
\hline \multicolumn{5}{|l|}{ Sexual behavior } \\
\hline Median number of sex partners in $<6$ mo (IQR) $\uparrow$ & $15(6.5-30)$ & $6(3-10)$ & $<0.001$ & $6(4-15)$ \\
\hline \multicolumn{5}{|l|}{ No. sex partners in $<6$ mo $\ddagger$} \\
\hline$\leq 3$ & $66(7.6)$ & $1124(27.7)$ & $<0.001$ & $1190(24.2)$ \\
\hline $4-6$ & $150(17.3)$ & $1150(28.4)$ & & $1300(26.4)$ \\
\hline $7-15$ & $282(32.6)$ & $1100(27.1)$ & & $1382(28.1)$ \\
\hline$\geq 16$ & $367(42.4)$ & $679(16.8)$ & & $1046(21.3)$ \\
\hline CAS $\S$ & $726(84.3)$ & $2461(61.1)$ & $<0.001$ & $3187(65.2)$ \\
\hline \multicolumn{5}{|l|}{ STI diagnoses } \\
\hline Any bacterial STI & $269(31.1)$ & $839(20.7)$ & $<0.001$ & $1108(22.5)$ \\
\hline Chlamydia (non-LGV) & $110(12.7)$ & $378(9.3)$ & 0.002 & $488(9.9)$ \\
\hline Pharyngeal & $14(1.6)$ & $45(1.1)$ & 0.212 & $59(1.2)$ \\
\hline Rectal & $97(11.2)$ & $290(7.1)$ & $<0.001$ & $387(7.9)$ \\
\hline Urethral & $42(4.8)$ & $134(3.3)$ & 0.026 & $176(3.6)$ \\
\hline LGV & $16(1.8)$ & $20(0.5)$ & $<0.001$ & $36(0.7)$ \\
\hline Gonorrhea & $165(19.1)$ & $445(11.0)$ & $<0.001$ & $610(12.4)$ \\
\hline Pharyngeal & $81(9.4)$ & $248(6.1)$ & 0.001 & $329(6.7)$ \\
\hline Rectal & $115(13.3)$ & $267(6.6)$ & $<0.001$ & $382(7.8)$ \\
\hline Urethral & $42(4.8)$ & $103(2.5)$ & $<0.001$ & $145(2.9)$ \\
\hline NSU\| & $27(3.1)$ & $127(3.1)$ & 0.986 & $154(3.1)$ \\
\hline Infectious syphilis & $37(4.3)$ & $109(2.7)$ & 0.012 & $146(3.0)$ \\
\hline New HIV diagnosis** & 2/570 (0.4) & 27/3432 (0.7) & 0.256 & 29/4002 (0.7) \\
\hline On PrEP $\uparrow \dagger$ & $145 / 568(25.5)$ & $195 / 3406(5.7)$ & $<0.001$ & $340 / 3974(8.6)$ \\
\hline
\end{tabular}

*Ethnicity was defined according to Statistics Netherlands on the basis of country of birth, and maternal and paternal country of birth. ${ }^{18}$

$\dagger$ HIV status also includes results of the current HIV test. MSM who refused an HIV test $(n=15)$ were added to the HIV-negative group.

\$Missing number of sex partners is $n=7$.

§CAS: no or inconsistent condom use or condom failure during anal sex. Missing number of CAS is $n=35$.

IBacterial STI: diagnosis with chlamydia, gonorrhea, LGV, and/or infectious syphilis.

|In those with more than 10 polymorph nucleated leucocytes per high-power field in a urethral Gram-stained smear and no infection with $\mathrm{Ct}$ and $\mathrm{Ng}$, a definitive diagnosis of NSU was made.

$* *$ Only tested in MSM not previously tested HIV positive.

$\dagger \dagger$ HIV-negative participants who used PrEP as part of the Amsterdam PrEP research study.

in chemsex and those not. The use of GHB during sex was most reported, with $93.0 \%$ in the past 6 months. Crystal methamphetamine use was reported by $22.1 \%$. Moreover, $13.0 \%$ of the MSM who engaged in chemsex did not have sober sex in the last 3 months and $6.1 \%$ stated injecting drugs.

Previous studies have shown that MSM who practiced chemsex exhibit high-risk behavior. ${ }^{4,11,16}$ In accordance with these findings, we found that MSM who engaged in chemsex show higher risk-taking behavior (more sex partners and more often CAS) compared with MSM who did not engage in chemsex. The sole use of GHB was reported by $68.1 \%$, and both crystal methamphetamine and GHB was the combination most used $(11.8 \%)$. Polydrug use as a predictor for risk behavior and STI has been previously reported. ${ }^{6}$ However, because we focused in our study only on the use of 3 drugs (crystal methamphetamine, $\mathrm{GHB}$, and mephedrone) during sex, we have no further knowledge of the participants' additional drug use. This might be a potential confounder to our results, because drugs have diverse mechanisms of actions that might lead to different risk behavior. ${ }^{21}$

Surprisingly, our findings revealed-irrespectively of chemsex practices - a relative small number of newly diagnosed HIV cases $(0.4 \%$ in chemsex group vs. $0.7 \% ; P=0.256)$. On the basis of previous findings, ${ }^{6,8,11}$ we expected a higher prevalence of new HIV diagnoses in the chemsex group. However, possible explanations for our results might be the short study period and that $25.5 \%$ of all MSM engaging in chemsex were using PrEP. One of the strengths of our study is the routinely collected detailed epidemiologic and behavioral data of clients of the STI 
TABLE 2. Univariable and Multivariable Analyses of Determinants Associated with a Bacterial STI* Diagnosis Among 4925 MSM, Attending the STI Clinic, Public Health Service of Amsterdam, the Netherlands; July to December 2016

\begin{tabular}{|c|c|c|c|c|c|c|}
\hline & $\begin{array}{c}\text { Total }(\mathrm{n}=4925), \\
\text { n (\% Within Group) }\end{array}$ & $\begin{array}{l}\text { Univariable, } \\
\text { OR }(95 \% \text { CI) }\end{array}$ & $\boldsymbol{P}$ & & $\begin{array}{l}\text { Multivariable } \dagger \text {, } \\
\text { aOR }(95 \% \text { CI) }\end{array}$ & $\boldsymbol{P}$ \\
\hline Age, y & & & 0.012 & & & $<0.001$ \\
\hline$\leq 27$ & $330 / 1379(23.9)$ & 1 & & & 1 & \\
\hline $28-35$ & $283 / 1208(23.4)$ & $1.0(0.8-1.2)$ & & & $0.8(0.7-1.0)$ & \\
\hline $36-46$ & $281 / 1207(23.3)$ & $1.0(0.8-1.2)$ & & & $0.7(0.6-0.9)$ & \\
\hline$\geq 47$ & $214 / 1131(18.9)$ & $0.7(0.6-0.9)$ & & & $0.5(0.4-0.6)$ & \\
\hline Ethnicity & & & 0.087 & & & \\
\hline Western & $706 / 3244(21.8)$ & 1 & & & & \\
\hline Non-Western & 402/1681 (23.9) & $1.1(1.0-1.3)$ & & & & \\
\hline Chemsex $<6$ mo & & & $<0.001$ & & & \\
\hline Not using chems during sex & $839 / 4059(20.7)$ & 1 & & & & \\
\hline Using chems during sex & $269 / 866(31.1)$ & $1.7(1.5-2.0)$ & & & & \\
\hline HIV status $\ddagger$ & & & $<0.001$ & & & \\
\hline HIV-negative & 794/3974 (20.0) & 1 & & & & \\
\hline HIV-positive & $314 / 951(33.0)$ & $2.0(1.7-2.3)$ & & & & \\
\hline Interaction chemsex $\times$ HIV status $\S$ & & & $<0.001$ & & & $<0.001$ \\
\hline No chemsex: HIV- & $628 / 3406(18.4)$ & 1 & & No chemsex: HIV- & 1 & \\
\hline No chemsex: HIV+ & $211 / 653(32.3)$ & $2.1(1.8-2.5)$ & & No chemsex: HIV+ & $2.0(1.6-2.5)$ & \\
\hline Yes chemsex: HIV- & $166 / 568(29.2)$ & $1.8(1.5-2.2)$ & & Yes chemsex: HIV- & $1.5(1.2-1.8)$ & \\
\hline Yes chemsex: HIV+ & $103 / 298(34.6)$ & $2.3(1.8-3.0)$ & & Yes chemsex: HIV+ & $1.8(1.3-2.3)$ & \\
\hline \multicolumn{7}{|l|}{ Reason of visit } \\
\hline Not notified of STI exposure & $742 / 3781(19.6)$ & 1 & $<0.001$ & & 1 & $<0.001$ \\
\hline Notification of STI exposure & $366 / 1144(32.0)$ & $1.9(1.7-2.2)$ & & & $2.0(1.7-2.3)$ & \\
\hline No STI-related symptoms & $686 / 3838(17.9)$ & 1 & $<0.001$ & & 1 & $<0.001$ \\
\hline STI-related symptoms & $422 / 1087(38.8)$ & $2.9(2.5-3.4)$ & & & $3.0(2.5-3.4)$ & \\
\hline $\mathrm{CAS}<6 \mathrm{mo}$ & & & $<0.001$ & & & $<0.001$ \\
\hline No CAS & $287 / 1703(16.9)$ & 1 & & & 1 & \\
\hline CAS & $813 / 3187(25.5)$ & $1.7(1.5-2.0)$ & & & $1.4(1.2-1.7)$ & \\
\hline No. sex partners $<6 \mathrm{mo} \|$ & & & $<0.001$ & & & $<0.001$ \\
\hline$\leq 3$ & 218/1190 (18.3) & 1 & & & 1 & \\
\hline $4-6$ & $274 / 1300(21.1)$ & $1.2(1.0-1.5)$ & & & $1.2(1.0-1.5)$ & \\
\hline $7-15$ & $312 / 1382(22.6)$ & $1.3(1.1-1.6)$ & & & $1.3(1.1-1.6)$ & \\
\hline$\geq 16$ & $302 / 1046(28.9)$ & $1.8(1.5-2.2)$ & & & $1.8(1.5-2.3)$ & \\
\hline
\end{tabular}

*Bacterial STI: diagnosis with chlamydia, gonorrhea, LGV, and/or infectious syphilis.

$\dagger$ In multivariable model, 4883 MSM were included, of whom 1108 had a bacterial STI.

$\$$ MSM who refused an HIV test were added to the HIV-negative group $(\mathrm{n}=15)$.

$\S$ The interaction between chemsex and HIV status for any bacterial STI was included in the multivariable model as a dummy variable with 4 categories.

qMissing number of CAS is $n=35$. No CAS: always condom use during anal sex or no anal sex. CAS: no or inconsistent condom use or condom failure during anal sex.

$\|$ Missing number of sex partners is $n=7$.

aOR indicates adjusted odds ratio.

clinic. Moreover, by comparing the STI clinic client's pattern of chemsex with users of a gay dating app, we showed that GHB and crystal methamphetamine use during sex is representative to a wider MSM population. However, caution should be taken in generalizing these findings because no demographics or risktaking behavior of the gay dating app users was available. Moreover, because the clinic population demonstrates already care-seeking behavior by visiting the clinic, it can be suggested that the overall chemsex engagement is even greater than what our clinic MSM population revealed. Also important to notice is that, to our knowledge, the present study is one of the first studies to show that chemsex disclosure is a predictor for a bacterial STI among HIV-negative MSM.

However, our study has some limitations. We do not have knowledge of the intentions for drug use, and the cross-sectional design does not allow for causal explanations. Our findings show that chemsex engagement and STI diagnoses are associated with each other in HIV-negative MSM, although we cannot attribute a causal relationship of chemsex to contracting STI.

Another limitation of our study might be the representativeness of the study population to the general MSM population.
We could only estimate associations between chemsex and STI diagnoses in clients of the STI clinic, who are explicitly seeking STI care. However, previous studies already have shown that chemsex engagement in different MSM populations was associated with high-risk behavior. ${ }^{8,11,12}$ Lastly, because we did not ask the dosage of the drugs they used during sex, we could not examine doseresponse relations.

This study revealed that especially among HIV-negative MSM, chemsex engagement seems an attributable risk factor for a bacterial STI diagnosis. In contrast, among the total group of HIV-positive MSM, the risk on an STI diagnosis is increased, irrespective of chemsex engagement. Likewise, Bourne et al. ${ }^{8}$ described that the HIV-positive men in their study did not attribute sexual risk behavior to chemsex, in contrast to HIV-negative men who perceived that drug use directly influenced unintentional sexual risk behavior. Therefore, future STI prevention strategies and health promotion should especially target HIV-negative men engaging in chemsex besides HIV-positive men.

Future research should have a cohort design to monitor change in chemsex over time and study causal relations. Moreover, data on chemsex-related drug dependence, the perception 
TABLE 3. Pattern of Chemsex and Drugs Used Among 866 MSM, at the STI Clinic, Public Health Service of Amsterdam, the Netherlands (July-December 2016) and 537 MSM Visitors of an Online Dating App

\begin{tabular}{|c|c|c|c|c|}
\hline \multirow[b]{2}{*}{ Chemsex, Yes } & \multirow{2}{*}{$\begin{array}{c}\frac{866 / 4925(17.6)}{\text { STI Clinic }} \\
(n=866), n(\%)\end{array}$} & \multirow{2}{*}{$\begin{array}{l}537 / 1832(29.3) \\
\text { Online Survey } \\
(n=537), n(\%)\end{array}$} & \multirow{2}{*}{$\begin{array}{c}<0.001 \\
P\end{array}$} & \multirow{2}{*}{$\begin{array}{c}\frac{1403 / 6757(20.8)}{\text { Total }} \\
(n=1403), n(\%)\end{array}$} \\
\hline & & & & \\
\hline GHB (G) use during sex $<6 \mathrm{mo}^{* \dagger} \uparrow$ yes & $798(93.0)$ & $453(91.1)$ & 0.215 & $1251(92.3)$ \\
\hline Once per month or less & $500(62.7)$ & $263(58.1)$ & 0.173 & $763(61.0)$ \\
\hline $2-4$ per month & $266(33.3)$ & $163(36.0)$ & & $429(34.3)$ \\
\hline $2-3$ a week & $27(3.4)$ & $20(4.4)$ & & $47(3.8)$ \\
\hline$\geq 4$ a week & $5(0.6)$ & $7(1.5)$ & & $12(1.0)$ \\
\hline Crystal methamphetamine (T) use during sex $<6 \mathrm{mo}^{*}+$, yes & $190(22.1)$ & $107(24.9)$ & 0.271 & $297(23.1)$ \\
\hline Once per month or less & $143(75.3)$ & $84(78.5)$ & 0.448 & $227(76.4)$ \\
\hline $2-4$ per month & $39(20.5)$ & $17(15.9)$ & & $56(18.9)$ \\
\hline $2-3$ a week & $6(3.2)$ & $6(5.6)$ & & $12(4.0)$ \\
\hline$\geq 4$ a week & $2(1.1)$ & 0 & & $2(0.7)$ \\
\hline Mephedrone (M) use during sex $<6$ mo, yes* $\S$ & $137(16.0)$ & $121(28.3)$ & $<0.001$ & $258(20.1)$ \\
\hline Once per month or less & $113(82.5)$ & $94(77.7)$ & 0.649 & $207(80.2)$ \\
\hline $2-4$ per month & $22(16.1)$ & $26(21.5)$ & & $48(18.6)$ \\
\hline $2-3$ a week & $1(0.7)$ & $1(0.8)$ & & $2(0.8)$ \\
\hline$\geq 4$ a week & $1(0.7)$ & 0 & & $1(0.4)$ \\
\hline Single/polydrug use during sex $<6 \mathrm{mo}^{* \mathrm{q}}$ & & & 0.008 & \\
\hline G only & $584(68.1)$ & $313(63.4)$ & & $897(66.3)$ \\
\hline $\mathrm{G}$ and $\mathrm{T}$ & $101(11.8)$ & $42(8.5)$ & & $143(10.6)$ \\
\hline $\mathrm{G}$ and $\mathrm{M}$ & $63(7.3)$ & $56(11.3)$ & & $119(8.8)$ \\
\hline $\mathrm{T}$ and $\mathrm{G}$ and $\mathrm{M}$ & $50(5.8)$ & $42(8.5)$ & & $92(6.8)$ \\
\hline T only & $36(4.2)$ & $18(3.6)$ & & $54(4.0)$ \\
\hline M only & $21(2.4)$ & $18(3.6)$ & & $39(2.9)$ \\
\hline $\mathrm{T}$ and $\mathrm{M}$ & $3(0.3)$ & $5(1.0)$ & & $8(0.6)$ \\
\hline \multicolumn{5}{|l|}{ Injecting drugs $<6 \mathrm{mo} \|$} \\
\hline Yes & $52(6.1)$ & $38(7.4)$ & 0.332 & $90(6.6)$ \\
\hline Shared needles & $1 / 52(1.9)$ & $4 / 38(10.5)$ & 0.078 & $5 / 90(5.6)$ \\
\hline Sober sex $* *$ & & & $<0.001$ & \\
\hline In the past month & $751(87.0)$ & $393(76.8)$ & & $1144(83.2)$ \\
\hline Longer than 3 mo ago & $47(5.5)$ & $43(8.4)$ & & $90(6.5)$ \\
\hline Longer than 6 mo ago & $14(1.6)$ & $14(2.7)$ & & $28(2.0)$ \\
\hline Longer than $1 \mathrm{y}$ ago & $31(3.6)$ & $30(5.9)$ & & $61(4.4)$ \\
\hline I do not remember & $20(2.3)$ & $32(6.3)$ & & $52(3.8)$ \\
\hline Condomless anal chemsex $\dagger \dagger$, yes & $600(69.8)$ & $317(62.4)$ & 0.005 & $917(67.1)$ \\
\hline
\end{tabular}

*Missing number of GHB, crystal methamphetamine, and mephedrone use is $\mathrm{n}=8$ (STI clinic).

$\dagger$ Missing number of GHB use is $n=40$ (online survey).

$\$$ Missing number of crystal methamphetamine use is $n=107$ (online survey).

$\S$ Missing number of mephedrone use is $\mathrm{n}=109$ (online survey).

qMissing number of GHB, crystal methamphetamine, and mephedrone use is $\mathrm{n}=43$ (online survey).

|Injecting 1 of the 3 drugs; missing numbers of injecting drugs are $n=7$ (STI clinic) and $n=23$ (online survey).

**Sex without the 3 drugs; missing numbers of sober sex are $\mathrm{n}=3$ (STI clinic) and $\mathrm{n}=25$ (online survey).

$\dagger \dagger$ CAS under the influence of 1 of the 3 drugs; missing numbers of condom use during chemsex are $n=7$ (STI clinic) and $n=29$ (online survey).

of whether chemsex engagement is personally perceived as a problem, and the impact on users' well-being are required.

Our study revealed that $87.0 \%$ of all MSM engaging in chemsex reported sober sex in the past month, indicating that dependency might not be an issue. Those findings are in agreement with Holt, ${ }^{22}$ who commented in The Lancet HIV that we should not forget that most men who engage in sex and drugs "do not experience harm and are not a threat to others." Conversely, Stuart et al. ${ }^{16}$ found that $70 \%$ of the MSM of their study population reported no chem-fee sex in the previous 6 months.

We are in the early stages of fully understanding the impact of the uprising phenomenon of chemsex, and it remains to be seen whether we are at a start of an emerging epidemic. Nonetheless, the present results show that there is a group of MSM in Amsterdam engaging in chemsex and that especially the HIV-negative men are at higher risk for STIs and potentially for HIV in the future. Therefore, the STI clinic of Amsterdam implemented a low threshold community-led counseling service for MSM who want to discuss chemsex and underlying motivations. ${ }^{7}$ If desired, referral to professional substance use and psychological care is possible.

\section{REFERENCES}

1. Stall R, Paul JP, Greenwood G, et al. Alcohol use, drug use and alcohol-related problems among men who have sex with men: The Urban Men's Health Study. Addiction 2001; 96:1589-1601.

2. Bourne A, Reid D, Hickson F, et al. The Chemsex Study: Drug Use in Sexual Settings Among Gay and Bisexual Men in Lambeth, Southwark and Lewisham. London: Sigma Research, 2014.

3. Schmidt AJ, Bourne A, Weatherburn P, et al. Illicit drug use among gay and bisexual men in 44 cities: Findings from the European MSM Internet Survey (EMIS). Int J Drug Policy 2016; 38: $4-12$.

4. Vosburgh HW, Mansergh G, Sullivan PS, et al. A review of the literature on event-level substance use and sexual risk behavior among men who have sex with men. AIDS Behav 2012; 16:1394-1410.

5. Heiligenberg M, Wermeling PR, van Rooijen MS, et al. Recreational drug use during sex and sexually transmitted infections among clients 
of a city sexually transmitted infections clinic in Amsterdam, the Netherlands. Sex Transm Dis 2012; 39:518-527.

6. Daskalopoulou M, Rodger A, Phillips AN, et al. Recreational drug use, polydrug use, and sexual behaviour in HIV-diagnosed men who have sex with men in the UK: Results from the cross-sectional ASTRA study. Lancet HIV 2014; 1:e22-e31.

7. Stuart D. A chemsex crucible: the context and the controversy. J Fam Plann Reprod Health Care 2016; 42:295-296.

8. Bourne A, Reid D, Hickson F, et al. Illicit drug use in sexual settings ('chemsex') and HIV/STI transmission risk behaviour among gay men in South London: Findings from a qualitative study. Sex Transm Infect 2015; 91:564-568.

9. Weatherburn P, Hickson F, Reid D, et al. Motivations and values associated with combining sex and illicit drugs ('chemsex') among gay men in South London: Findings from a qualitative study. Sex Transm Infect 2016.

10. Pakianathan MR, Lee MJ, Kelly B, et al. How to assess gay, bisexual and other men who have sex with men for chemsex. Sex Transm Infect 2016; 92:568-570.

11. Hegazi A, Lee MJ, Whittaker W, et al. Chemsex and the city: Sexualised substance use in gay bisexual and other men who have sex with men attending sexual health clinics. Int J STD AIDS 2017; 28:362-366.

12. Melendez-Torres GJ, Bourne A. Illicit drug use and its association with sexual risk behaviour among MSM: More questions than answers? Curr Opin Infect Dis 2016; 29:58-63.

13. Ma R, Perera S. Safer 'chemsex': GPs' role in harm reduction for emerging forms of recreational drug use. Br J Gen Pract 2016; 66:4-5.

14. Macfarlane A. Sex, drugs and self-control: Why chemsex is fast becoming a public health concern. J Fam Plann Reprod Health Care 2016; 42:291-294.
15. Knoops L, Bakker I, van Bodegom R, et al. Tina \& slammen: MSM, crystal meth-gebruik en het injecteren van drugs in een seksuele setting. Amsterdam: Mainline, Soa Aids Nederland, 2015.

16. Stuart D, Nwokolo N, McOwan A, et al. ChemSex: Data on recreational drug use and sexual behaviour in men who have sex with men (MSM) from a busy sexual health clinic in London, UK. Paper presented at: 15th European AIDS Conference; October 21-24, 2015; Barcelona.

17. van den Broek AJ, van Rooijen MS, van Veen MG, et al. Year report STI clinic Amsterdam 2014 (in Dutch, summary in English). 2014.

18. Alders M. Classification of the population with foreign background in the Netherlands, Statistics Netherlands. In: The Measure and Mismeasure of Populations: The Statistical Use of Ethnic and Racial Categories in Multicultural Societies. Paper presented at: CERI-INED, December 17-18, 2001; Paris.

19. Bartelsman M, van Rooijen MS, Alba S, et al. Point-of-care management of urogenital Chlamydia trachomatis via Gram-stained smear analysis in male high-risk patients. Diagnostic accuracy and cost-effectiveness before and after changing the screening indication at the STI clinic in Amsterdam. Sex Transm Infect 2015; 91 : 479-484.

20. Hoornenborg E, Achterbergh R, Schim van der Loeff M, et al. AMPrEP Team, Part of the HIV Transmission Elimination Amsterdam (H-TEAM) Consortium 2016.

21. Drumright LN, Patterson TL, Strathdee SA. Club drugs as causal risk factors for HIV acquisition among men who have sex with men: A review. Subst Use Misuse 2006; 41:1551-1601.

22. Holt M. Sex, drugs, and HIV: Let's avoid panic. Lancet HIV 2014 $1: e 4-e 5$. 